\title{
Intraarticular Glucocorticoid Injection as Second-line Treatment for Lyme Arthritis in Children
}

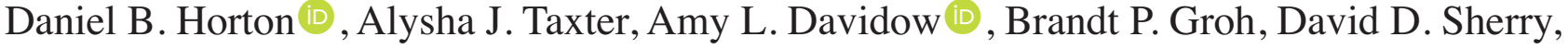 \\ and Carlos D. Rose
}

ABSTRACT. Objective. To determine whether second-line intraarticular glucocorticoid (IAGC) injection improves outcomes in children with persistently active Lyme arthritis after initial antibiotics.

Methods. We conducted an observational comparative effectiveness study through chart review within 3 pediatric rheumatology centers with distinct clinical approaches to second-line treatment of Lyme arthritis. We primarily compared children receiving second-line IAGC to children receiving a second course of antibiotics alone. We evaluated the risk of developing antibiotic-refractory Lyme arthritis (ARLA) using logistic regression and the time to clinical resolution of Lyme arthritis using Cox regression.

Results. Of 112 children with persistently active Lyme arthritis after first-line antibiotics, 18 children received second-line IAGC (13 with concomitant oral antibiotics). Compared to children receiving second-line oral antibiotics alone, children treated with IAGC had similar baseline characteristics but lower rates of ARLA (17\% vs 44\%; OR 0.3, 95\% CI 0.1-0.95; p = 0.04) and faster rates of clinical resolution (HR 2.2, 95\% CI 1.2-3.9; $\mathrm{p}=0.01$ ). Children in IAGC and oral antibiotic cohorts did not differ in treatment-associated adverse events. Among children receiving second-line IAGC, outcomes appeared similar irrespective of use of concomitant antibiotics. Outcomes were also similar between intravenous (IV) and oral antibiotic-treated cohorts, but older children seemed to respond more favorably to IV therapy. IV antibiotics were also associated with higher rates of toxicity.

Conclusion. IAGC injection appears to be an effective and safe second-line strategy for persistent Lyme arthritis in children, associated with rapid clinical resolution and reduced need for additional treatment. (First Release June 1 2019; J Rheumatol 2019;46:952-9; doi:10.3899/jrheum.180829)

Key Indexing Terms:

LYME ARTHRITIS

PEDIATRICS

COMPARATIVE EFFECTIVENESS RESEARCH

GLUCOCORTICOIDS EPIDEMIOLOGIC STUDIES

From the Division of Pediatric Rheumatology, Rutgers Robert Wood Johnson Medical School, New Brunswick; Rutgers Center for Pharmacoepidemiology and Treatment Science, Institute for Health, Health Care Policy and Aging Research, New Brunswick; Rutgers School of Public Health, Piscataway; Rutgers School of Public Health, Newark, New Jersey; Brenner Children's Hospital, Wake Forest Baptist Medical Center, Winston-Salem, North Carolina; Penn State Milton S. Hershey Medical Center, Hershey; Children's Hospital of Philadelphia, Division of Pediatric Rheumatology, Perelman School of Medicine, University of Pennsylvania, Philadelphia, Pennsylvania; Division of Rheumatology, Nemours/A.I. duPont Hospital for Children, Thomas Jefferson University, Wilmington, Delaware, USA.

This study was supported by the US National Institute of Arthritis and Musculoskeletal and Skin Diseases (NIAMS) of the National Institutes of Health (NIH) under Award Numbers F32-AR066461, L40-AR070497, and K23-AR070286, and the Eunice Kennedy Shriver National Institute of Child Health and Human Development of the NIH under Award Number T32-HD064567. The content is solely the responsibility of the authors and does not necessarily represent the official views of the NIH, NIAMS, or the Eunice Kennedy Shriver National Institute of Child Health and Human Development.

Dr. Rose has received grant funding from GSK via subcontract from the University of Leuven for research unrelated to the present study.

D.B. Horton, MD, MSCE, Division of Pediatric Rheumatology, Rutgers Robert Wood Johnson Medical School, and Rutgers Center for Pharmacoepidemiology and Treatment Science, Institute for Health, Health Care Policy and Aging Research, and Rutgers School of Public Health; A.J. Taxter, MD, MSCE, Brenner Children's Hospital, Wake Forest
Baptist Medical Center; A.L. Davidow, PhD, Rutgers School of Public Health; B.P. Groh, MD, Penn State Milton S. Hershey Medical Center; D.D. Sherry, MD, Children's Hospital of Philadelphia, Division of Pediatric Rheumatology, Perelman School of Medicine, University of Pennsylvania; C.D. Rose, MD, Division of Rheumatology, Nemours/ A.I. duPont Hospital for Children, Thomas Jefferson University. Address correspondence to Dr. D.B. Horton, 112 Paterson St., New Brunswick, New Jersey 08901,USA.E-mail: daniel.horton@rutgers.edu Accepted for publication December 18, 2018.

Lyme disease is the most common vector-borne illness in the United States and Europe, with rising incidence and expanding geographic distribution in some areas ${ }^{1,2,3}$. Lyme arthritis represents a late infectious complication, most commonly affecting the knee, caused by direct dissemination of spirochetes to the synovium and resultant synovitis ${ }^{4,5,6}$. While early stages of Lyme disease are generally cured with a single course of antibiotics, $10-30 \%$ of those with Lyme arthritis do not experience clinical resolution after a single course of antibiotics ${ }^{7,8}$. Unlike first-line treatment of Lyme arthritis, second-line regimens endorsed by current treatment guidelines are not supported by randomized trials or other high-quality evidence but instead reflect clinical experience and expert opinion ${ }^{9,10}$. 
Intraarticular glucocorticoids (IAGC) have been described as a treatment for antibiotic refractory Lyme arthritis (ARLA), a postinfectious inflammatory condition ${ }^{11,12}$. However, premature IAGC injection that precedes initial antibiotic therapy has been reported as a potential risk factor for the development of ARLA in children and adults ${ }^{11,13}$. This risk is attributed to impairment of the body's response to infection due to local immune suppression within an infected joint. Some centers have used IAGC as a standard component of second-line treatment for patients who do not respond to a first course of antibiotics. We sought to exploit real-world variation in clinical practice to determine whether IAGC with or without second-line antibiotic therapy hastens the resolution of Lyme arthritis and prevents the development of ARLA in children.

\section{MATERIALS AND METHODS}

Design. We conducted a retrospective cohort study within a larger cohort of children diagnosed with Lyme arthritis.

Setting. Participants were seen in 1 of 3 pediatric rheumatology clinics [Children's Hospital of Philadelphia (CHOP), Nemours/A.I. duPont Hospital for Children, and Penn State Hershey Children's Hospital] serving children from 4 Lyme-endemic US states (Delaware, New Jersey, Maryland, and Pennsylvania). In 1 center, second-line IAGC was the standard treatment for Lyme arthritis persisting after a single antibiotic course. Our study was approved by the Institutional Review Boards of the participating centers (CHOP IRB 14-010818, Nemours \#598679, Hershey STUDY00000431) and Rutgers University (PRO Pro20170002088) with a waiver of consent/assent for this minimal-risk retrospective research.

Study population. The study included children diagnosed with Lyme arthritis at age $\leq 18$ years, seen between January 1, 2000, and December 31, 2013, who received second-line therapy for persistently active Lyme arthritis within 120 days of starting first-line antibiotics. We defined active arthritis on examination as documentation by a physician of joint effusion or 2 other inflammatory signs (warmth, tenderness, restricted or painful range of motion). Initiation of second-line treatment beyond the 120-day window was atypical in the 3 centers and due to reasons unrelated to second-line treatment selection (e.g., inconsistent followup, recurrent arthritis); therefore, we excluded patients with such delays in treatment to represent usual care. We screened for participants in electronic health records (EHR) based on a diagnosis of Lyme disease (International Classification of Diseases, 9th revision, Clinical Modification code 088.81) and reviewed records to confirm Lyme arthritis diagnosis using these criteria: (1) documented positive Lyme testing with $\geq 5 \mathrm{IgG}$ bands on Western blot performed with standard methods; (2) documented arthritis by physical examination or arthrocentesis; and (3) exclusion of other causes of arthritis. Physicians at participating centers routinely diagnosed children with Lyme arthritis despite the occasional omission of Lyme screening by ELISA as recommended by guidelines ${ }^{9,10}$. Children with missing Lyme ELISA results $(\mathrm{n}=22)$ were excluded from sensitivity analyses (see statistical analysis).

Definitions of exposure. The primary exposure group of interest received second-line IAGC with or without concomitant antibiotics [referred to herein as $\mathrm{C}$ (cohort)-GC]. Primary comparators received a second course of oral antibiotics without accompanying IAGC (C-PO). Secondarily, we compared children receiving second-line intravenous (IV) antibiotics (C-IV) to C-PO. To compare the experimental use of second-line IAGC with accepted clinical practices, we required children in C-PO and C-IV to receive guideline-compatible treatments, with $\geq 8$ weeks of oral antibiotics (doxycycline, amoxicillin, or cefuroxime) or $\geq 2$ weeks of IV antibiotics (ceftriaxone or cefotaxime $)^{9,10}$ and the second course beginning within 120 days of antibiotic initiation. We required children in C-GC to receive $\geq 4$ weeks of oral antibiotics.
Outcomes. The primary outcome was development of ARLA, defined as documentation of persistently active Lyme arthritis $\geq 2$ months after completing $\geq 8$ weeks of oral antibiotics (amoxicillin, doxycycline, or cefuroxime) or $\geq 2$ weeks of IV antibiotics (ceftriaxone or cefotaxime); if performed, Lyme PCR testing of synovial fluid was negative ${ }^{9}$. Among children in C-GC without a second course of antibiotics, we also classified as ARLA having persistently active arthritis $\geq 3$ months after second-line IAGC. Sensitivity analyses considered a secondary outcome of persistently active arthritis for $\geq 6$ months after initiating first-line antibiotics. We defined clinical resolution as having all symptoms resolved and no more than a small joint effusion. Small, asymptomatic effusions were deemed compatible with clinical resolution because (1) joint swelling from Lyme arthritis can dissipate slowly after eradication of infection, and (2) physicians in all 3 centers routinely stopped treating and following patients in this clinical state. Covariates. We considered a range of potential confounders that might independently relate to treatment selection and risk of ARLA, including demographics, clinical presentation, and course before second-line treatment, and initial treatment (Table 1$)^{14}$. We characterized trajectory of disease activity based on whether there was improvement, worsening, or no change in clinical condition (i.e., symptoms or signs of arthritis) leading up to second-line treatment. We defined marked clinical worsening as having newly developed a massive effusion, popliteal cyst rupture, or symptomatic joint recruitment after antibiotic initiation.

Data collection. We abstracted data from EHR using standardized forms on Research Electronic Data Capture tools hosted at Nemours ${ }^{15}$. Data collection was performed by attending pediatric rheumatologists, pediatric rheumatology fellows with $\geq 1$ year of clinical training, or trained research staff; supervising physicians reviewed charts abstracted by nonclinical research staff to confirm accuracy. Inconsistent data triggered additional EHR review and data revision.

Statistical analysis. We described cohorts using standard descriptive statistics. The primary analysis used logistic regression to estimate the association between second-line treatment and ARLA. Multivariable models incorporated confounding variables that changed the OR between C-GC and C-PO by $\geq 10 \%$. Secondary analyses examined the association between second-line treatment and time to clinical resolution using Cox proportional hazards regression, adjusting for confounders. We examined the possibility of effect modification by age ( $<10 \mathrm{vs} \geq 10 \mathrm{yrs}$ ) and considered $\mathrm{p}<0.1$ by likelihood ratio testing of nested models suggestive of a significant interaction. Sensitivity analysis considered (1) additional, nonconfounding covariates in multivariable models, (2) alternate outcome definition, (3) exclusion for lack of Lyme antibody screening, (4) exclusion for IAGC without 2nd-line antibiotics, (5) exclusion of children who received second-line treatment within 4 weeks of starting first-line treatment, (6) exclusion of children who received second-line treatment within 8 weeks of starting first-line treatment, (7) exclusion of children lost to followup (logistic regression), (8) imputation of date of resolution among those lost to followup to estimate ARLA status (logistic regression), (9) worst-case imputation, where loss to followup was interpreted as treatment failure (logistic regression), or (10) analysis of time to resolution starting with second-line therapy (Cox regression). We considered variables with missing data such as arthritis duration to be missing at random, given their association with clinical center, outcome, and other variables ${ }^{14}$. We therefore used multiple imputation with 20 imputed data sets for missing data ${ }^{16}$.

We used Stata version 12.1 (StataCorp.) for all analyses and considered 2 -sided $\mathrm{p}$ values $<0.05$ significant.

\section{RESULTS}

Of 383 individuals with confirmed Lyme arthritis in the original cohort, we identified 112 eligible individuals who received second-line treatment for persistently active Lyme arthritis within 120 days (Figure 1). All IAGC in C-GC involved the knee (both knees in 1 subject). Children in C-GC

Personal non-commercial use only. The Journal of Rheumatology Copyright @ 2019 . All rights reserved. 
Table 1. Characteristics of subjects in study.

\begin{tabular}{|c|c|c|c|c|c|}
\hline Characteristics & $\mathrm{C}-\mathrm{PO}, \mathrm{n}=63$ & $\mathrm{C}-\mathrm{GC}, \mathrm{n}=18$ & $\mathrm{p}, \mathrm{C}-\mathrm{GC}$ vs C-PO & $\mathrm{C}-\mathrm{IV}, \mathrm{n}=31$ & $\mathrm{p}, \mathrm{C}-\mathrm{IV}$ vs C-PO \\
\hline Age, yrs, median (IQR; range) & $11(9-14 ; 2-16)$ & $11(8-15 ; 3-17)$ & $0.64^{\mathrm{b}}$ & $12(10-14 ; 5-17)$ & $0.78^{\mathrm{b}}$ \\
\hline Male sex & $45(71)$ & $11(61)$ & 0.40 & $17(55)$ & 0.11 \\
\hline \multicolumn{6}{|c|}{ Continuous joint symptoms at presentation } \\
\hline$\geq 6$ weeks & $9(14)$ & $2(11)$ & $0.60^{\mathrm{c}}$ & $3(10)$ & $0.31^{\mathrm{c}}$ \\
\hline Unknown & $18(29)$ & $3(17)$ & & $5(16)$ & \\
\hline Severe arthritis ${ }^{\mathrm{d}}$ & $22(35)$ & $8(44)$ & 0.46 & $11(35)$ & 0.96 \\
\hline Premature IAGC & $1(2)$ & $1(6)$ & $0.40^{\mathrm{c}}$ & 0 & $0.99^{\mathrm{c}}$ \\
\hline HLA-B27-positive & $3(5)$ & 0 & $0.42^{\mathrm{c}}$ & $1(3)$ & $0.56^{\mathrm{c}}$ \\
\hline HLA-B27 missing & $48(76)$ & $12(67)$ & & $27(87)$ & \\
\hline \multicolumn{6}{|l|}{ Dose of first antibiotic course } \\
\hline Correct per guidelines ${ }^{6,7}$ & $47(75)$ & $13(72)$ & $0.34^{\mathrm{c}}$ & $24(77)$ & $0.99^{c}$ \\
\hline Worsening & $18(29)$ & $3(17)$ & & $20(65)$ & \\
\hline $\begin{array}{l}\text { Marked clinical worsening } \leq 6 \text { weeks after } \\
\text { antibiotic initiation }^{f}\end{array}$ & $9(14)$ & 0 & $0.20^{\mathrm{c}}$ & $12(39)$ & 0.01 \\
\hline $\begin{array}{l}\text { Days of followup from diagnosis to final } \\
\text { rheumatology clinic visit }\end{array}$ & $263(155-580)$ & $102(74-232)$ & $<0.01^{\mathrm{b}}$ & $264(101-772)$ & 0.62 \\
\hline $\begin{array}{l}\text { Days of followup from diagnosis to final } \\
\text { encounter in EHR }\end{array}$ & $449(200-1026)$ & $331(100-1060)$ & 0.40 & $309(101-1084)$ & 0.35 \\
\hline
\end{tabular}

Values are median (IQR) or $\mathrm{n}(\%)$ unless otherwise specified. ${ }^{\text {a }} \mathrm{P}$ value calculated from chi-square testing except where indicated. ${ }^{\mathrm{b}} \mathrm{P}$ value calculated from Wilcoxon rank-sum testing. ${ }^{\mathrm{c}} \mathrm{P}$ value calculated from Fisher's exact test. ${ }^{\mathrm{d}}$ Unexplained fever, severe pain, hospitalization for severe pain, or measured sedimentation rate $\geq 40 \mathrm{~mm} / \mathrm{h}$. ${ }^{\text {e }}$ Early improvement with subsequent worsening by the time of second-line treatment was characterized as worsening; early worsening with subsequent improvement by the time of second-line treatment was characterized as improving; no change indicated no change in clinical activity (i.e., extent of synovitis) from the time of first-line treatment to second-line treatment. ${ }^{\mathrm{f}}$ Massive effusion, popliteal cyst rupture, or symptomatic joint recruitment after antibiotic initiation. C-GC: intraarticular glucocorticoid cohort; C-IV: intravenous antibiotics cohort; C-PO: oral antibiotics cohort; EHR: electronic health record; IAGC: intraarticular glucocorticoid; IQR: interquartile range.

did not significantly differ from children in C-PO in any measured baseline characteristics, including age, duration of joint symptom at diagnosis, pattern of joint disease, and trend of disease activity at initiation of second-line treatment, reflecting the use of second-line IAGC based on physician preferences rather than clinical features (Table 1). Of 18 children in C-GC, 13 (72\%) also received second-line oral antibiotics (Table 2). Compared with children in C-PO, children in C-IV were more likely to have early clinical worsening $(65 \%$ vs $29 \%, \mathrm{p}<0.01)$ and marked clinical worsening $(39 \%$ vs $14 \%, \mathrm{p}=0.01)$ on initial antibiotic therapy. As a result, children in C-IV more commonly started second-line treatment sooner after initial antibiotics [median 35 days (interquartile range; IQR 21-53) vs median 45 days (IQR 33-63), $\mathrm{p}=0.01$; Table 1 and Table 2].

Overall, 43/112 (38\%) children treated with second-line therapy developed ARLA. Only $17 \%$ (3/18) in C-GC were diagnosed with ARLA compared with 44\% (28/63) in C-PO (Table 3), translating into an OR for ARLA of 0.3 (95\% CI $0.1-0.95, p=0.04$ ). Rates of ARLA in C-GC were similar among those who did and did not receive a concomitant course of oral antibiotics [2/13 (15\%) and 1/5 (20\%), respec- tively]. Compared to C-PO, a similar proportion of those in C-IV $(12 / 31,39 \%)$ developed ARLA (OR 0.8, 95\% CI $0.3-1.9 ; \mathrm{p}=0.60)$. A time-to-event analysis showed that children in C-GC experienced resolution of arthritis at over twice the rate as children in C-PO (HR 2.2, 95\% CI 1.2-3.9; $\mathrm{p}=0.01$; Figure 2$)$. Rates of resolution did not significantly differ between C-IV and C-PO (HR 1.3, 95\% CI 0.8-2.1; $\mathrm{p}=0.30$ ). Inclusion of additional covariates in multivariable models did not appreciably change the results (i.e., there was no evidence of confounding) except for modest changes in the estimates for C-IV when modeling the trend of disease activity at second-line treatment initiation (Supplementary Tables 1 and 2, available with the online version of this article).

The median time from IAGC to documented resolution of Lyme arthritis was 43 days (IQR 35-80), corresponding to one center's standard 6-week followup after IAGC. In contrast, the median time to resolution after second-course oral antibiotics alone was 149 days (IQR 65-285); after IV antibiotics, 83 days (IQR 48-262; Table 3, Figure 3). Only 1 child $(6 \%)$ and 2 children $(11 \%)$ in $\mathrm{C}-\mathrm{GC}$ received subsequent IAGC or third-line antibiotic treatment, respectively.

Personal non-commercial use only. The Journal of Rheumatology Copyright @ 2019. All rights reserved. 


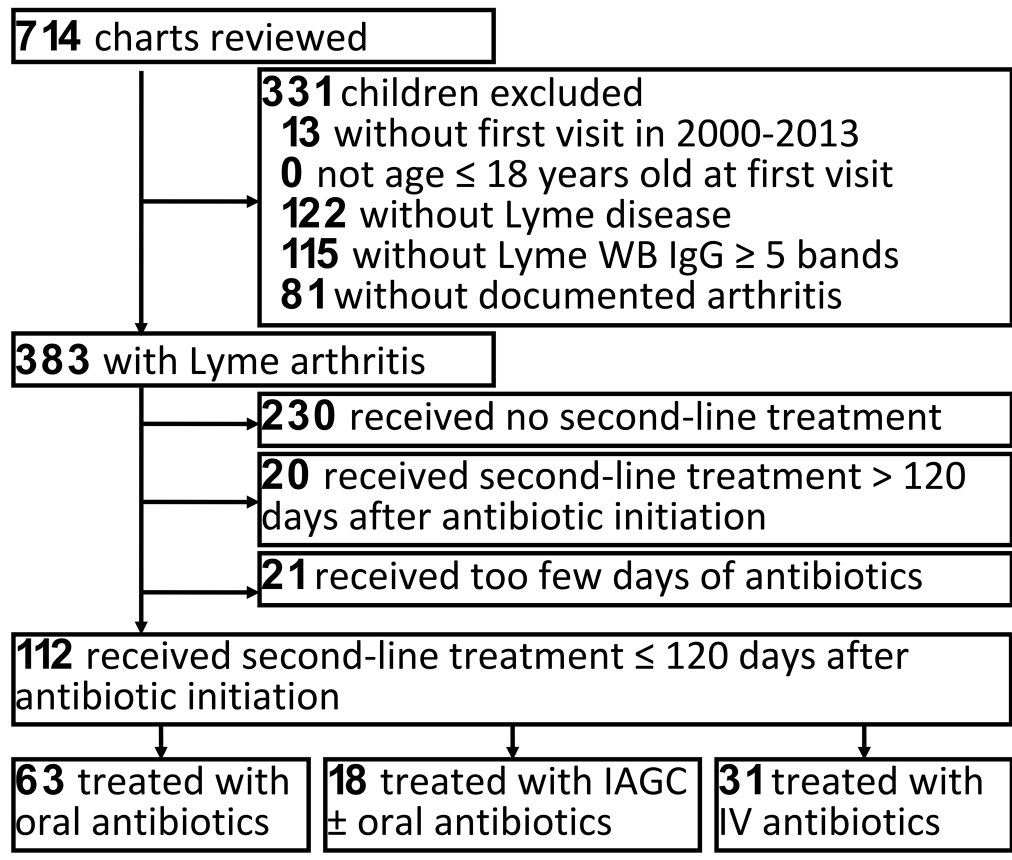

Figure 1. Subject selection diagram. Of 383 children with documented Lyme arthritis, 112 met inclusion criteria by beginning second-line treatment for persistent arthritis within 120 days after starting first-line antibiotics, of whom 18 individuals (16\%) received second-line IAGC injection. IAGC: intraarticular glucocorticoid; WB: Western blot; IgG: immunoglobulin G.

Table 2. Characteristics of second-line treatments.

\begin{tabular}{|c|c|c|c|c|c|}
\hline Characteristics & $\mathrm{C}-\mathrm{PO}, \mathrm{n}=63$ & $\mathrm{C}-\mathrm{GC}, \mathrm{n}=18$ & $\mathrm{p}, \mathrm{C}-\mathrm{GC}$ vs C-PO & $\mathrm{C}-\mathrm{IV}, \mathrm{n}=31$ & $\mathrm{p}, \mathrm{C}-\mathrm{IV}$ vs C-PO \\
\hline \multicolumn{6}{|l|}{ Second-line antibiotics, n (\%) } \\
\hline Doxycycline & $49(78)$ & $9(50)^{c}$ & $<0.01^{\mathrm{b}}$ & 0 & $<0.01^{\mathrm{b}}$ \\
\hline Cefuroxime & $2(3)$ & $1(6)$ & & 0 & \\
\hline Ceftriaxone & 0 & 0 & & $31(100)$ & \\
\hline None & 0 & $5(28)$ & & 0 & \\
\hline NSAID use with second-line therapy, n (\%) & $17(27)$ & $1(6)$ & $0.06^{\mathrm{b}}$ & $12(39)$ & 0.25 \\
\hline \multicolumn{6}{|c|}{ Positive synovial Lyme PCR test before second-line treatment, } \\
\hline $\mathrm{n}$ positive/tested & $0 / 2$ & $0 / 0$ & - & $2 / 4$ & - \\
\hline
\end{tabular}

${ }^{a} \mathrm{P}$ value calculated from chi-square testing except where indicated. ${ }^{\mathrm{b}} \mathrm{P}$ value calculated from Fisher's exact test. ${ }^{\mathrm{c}}$ Sum of percentages exceeds $100 \%$ because of rounding. ${ }^{\mathrm{d}} \mathrm{P}$ value calculated from Wilcoxon rank-sum testing. C-GC: intraarticular glucocorticoid cohort; C-IV: intravenous antibiotics cohort; C-PO: oral antibiotics cohort; IQR: interquartile range; NSAID: nonsteroidal antiinflammatory drug.

Rates of subsequent IAGC treatment for ARLA were higher in C-PO $(46 \%, \mathrm{p}<0.01)$ and C-IV $(26 \%, \mathrm{p}=0.04$ vs oral antibiotics). The 1 child in C-GC without second-line antibiotics who developed ARLA had received a premature IAGC before antibiotic initiation; among those without concomitant second-line antibiotics, this child was the only one who required subsequent antibiotics (oral followed by IV) or had a documented recurrence of arthritis after treatment.

We performed several analyses to better understand potential modifiers of treatment outcomes. When evaluating whether age modified the effect of IAGC, we found no differences in the risk of ARLA or rate of arthritis resolution between children under 10 years and children 10 years and older (Supplementary Table 3, available with the online version of this article). In contrast, age appeared to substantially modify the effects of IV antibiotics: children $<10$ years in C-IV appeared to have a high risk of developing ARLA $(5 / 6,83 \%)$ and slower rates of resolution [median 315 (IQR 148-525) days] compared to children $\geq 10$ years [ARLA 7/25, 28\%; median days to resolution 63 (IQR 48-114)]. Multivariable modeling suggested a significant treatment/age 
Table 3. Outcomes of interest by cohort.

\begin{tabular}{|c|c|c|c|c|c|}
\hline Outcomes & $\mathrm{C}-\mathrm{PO}, \mathrm{n}=63$ & $\mathrm{C}-\mathrm{GC}, \mathrm{n}=18$ & $\mathrm{p}, \mathrm{C}-\mathrm{GC}$ vs C-PO & $\mathrm{C}-\mathrm{IV}, \mathrm{n}=31$ & $\mathrm{p}, \mathrm{C}-\mathrm{IV}$ vs C-PO \\
\hline Development of ARLA & $28(44)$ & $3(17)$ & 0.03 & $12(39)$ & 0.60 \\
\hline \multicolumn{6}{|l|}{ Days from second-line antibiotic initiation to } \\
\hline Persistent arthritis at $6 \mathrm{mos}$ & $25(40)$ & $3(17)$ & 0.07 & $9(29)$ & 0.31 \\
\hline No. subsequent clinic visits & $3(2-5)$ & $1(1-2)$ & $<0.01^{\mathrm{b}}$ & $2(1-5)$ & $0.34^{\mathrm{b}}$ \\
\hline Subsequent antibiotic treatment & $17(27)$ & $2(11)$ & $0.22^{\mathrm{c}}$ & $1(3)$ & $<0.01$ \\
\hline 2 IAGC & $6(10)$ & $1(6)$ & & $3(10)$ & \\
\hline$\geq 3$ IAGC & $2(3)$ & 0 & & $2(6)$ & \\
\hline Subsequent DMARD use & $5(8)$ & 0 & $0.58^{\mathrm{c}}$ & $2(7)$ & $0.99^{\mathrm{c}}$ \\
\hline Subsequent synovectomy & $4(6)$ & $1(6)$ & $0.99^{\mathrm{c}}$ & $1(3)$ & $0.99^{\mathrm{c}}$ \\
\hline Lost to followup & $10(16)$ & $3(17)$ & $0.99^{\mathrm{c}}$ & $8(27)$ & 0.25 \\
\hline Lost to followup before ARLA determination & $3(5)$ & $3(17)$ & $0.12^{\mathrm{c}}$ & $5(16)$ & $0.11^{\mathrm{c}}$ \\
\hline
\end{tabular}

Values are $\mathrm{n}(\%)$ or median (IQR). ${ }^{\text {a }} \mathrm{P}$ value calculated from chi-square testing except where indicated. ${ }^{\mathrm{b}} \mathrm{P}$ value calculated from Wilcoxon rank-sum testing. ${ }^{c}$ P value calculated from Fisher's exact test. ARLA: antibiotic-refractory Lyme arthritis; C-GC: intraarticular glucocorticoid cohort; C-IV: intravenous antibiotics cohort; C-PO: oral antibiotics cohort; IAGC: intraarticular glucocorticoid; IQR: interquartile range; DMARD: disease-modifying antirheumatic drug.

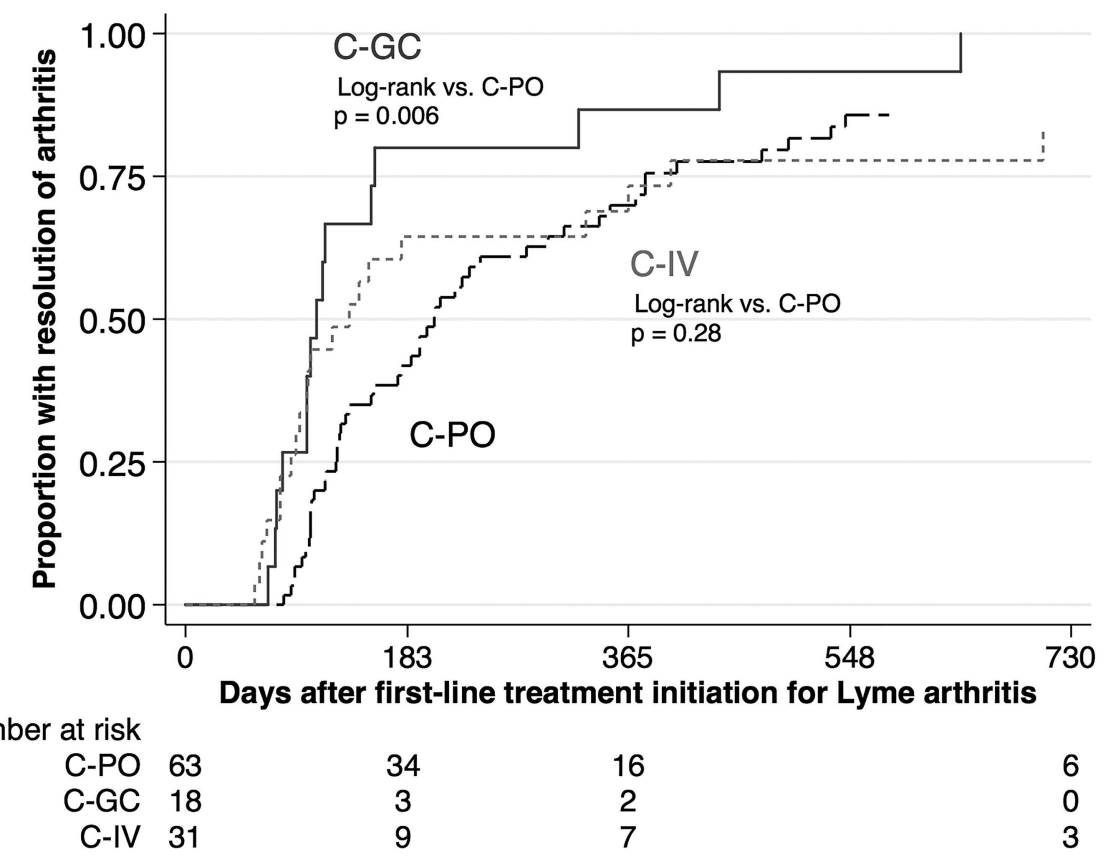

\begin{abstract}
Figure 2. Kaplan-Meier cumulative incidence curves of resolution of Lyme arthritis after initiating first-line antibiotics. Cumulative incidence plots showing resolution of Lyme arthritis over the first 2 years of followup by cohort: C-PO (black, long dash line), C-GC (dark grey, solid line), or C-IV (light grey, short dash line). Log-rank tests are shown comparing either C-GC or C-IV antibiotics to C-PO. C-GC: intraarticular glucocorticoid cohort; C-IV: intravenous antibiotics cohort; C-PO: oral antibiotics cohort.
\end{abstract}

interaction $(\mathrm{p}=0.05$ for ARLA risk, $\mathrm{p}=0.003$ for time to resolution) even though, given the small numbers in each age subgroup, the CI included the null for most age-stratified models (Supplementary Table 3). When focusing just on C-IV, we found few significant demographic or baseline clinical differences between children who did and did not respond to second-line ceftriaxone, except that responders appeared to have shorter duration of symptoms at diagnosis $(\mathrm{p}=0.01 ;$ Supplementary Table 4$)$. Additionally, aside from possible age-related differences within C-IV, children who responded to IV ceftriaxone appeared more likely to have marked worsening on initial treatment $(60 \%$ vs $23 \%, \mathrm{p}=0.1)$. 


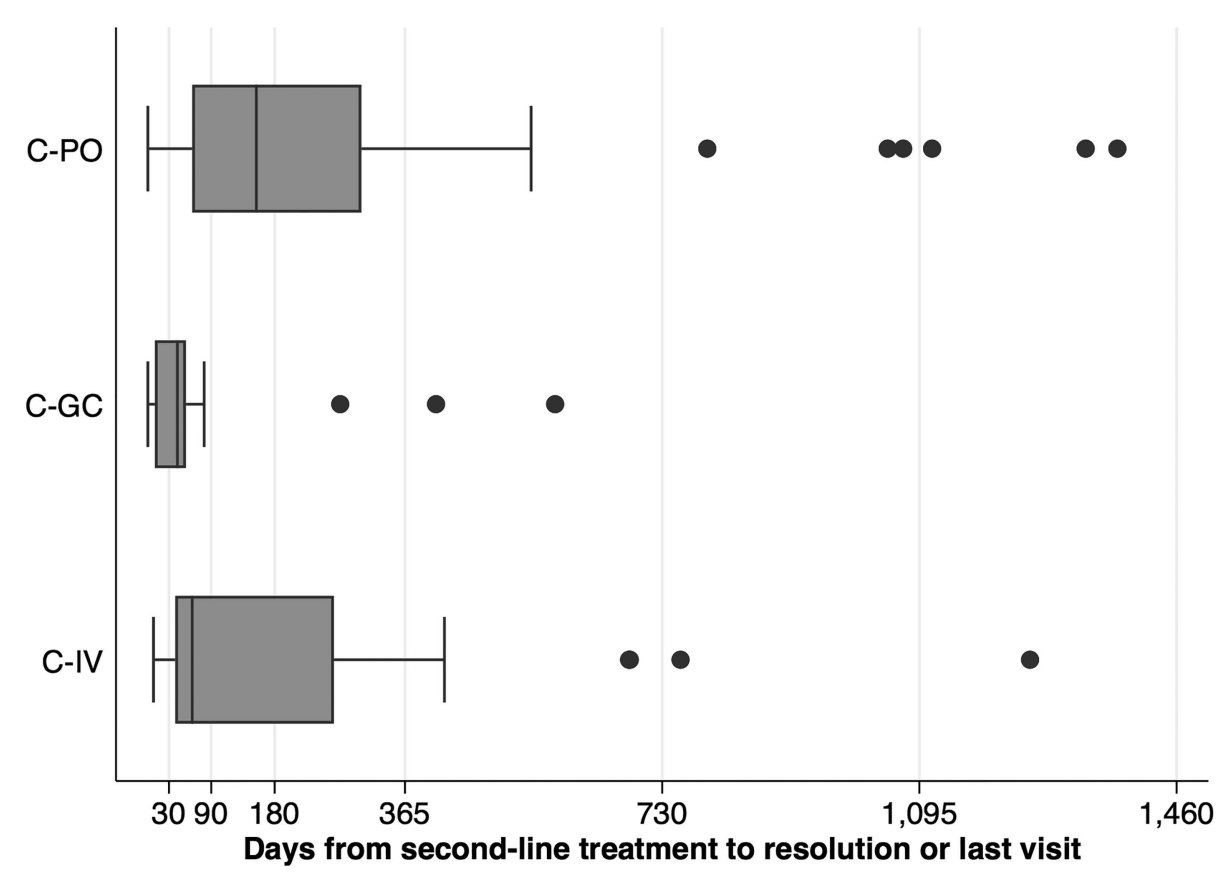

Figure 3. Timeline of outcomes among study subjects. Box plots showing, by treatment cohort, the timing from second-line treatment to the resolution of arthritis or the last visit (for those lost to followup). Boxes indicate the median (central bar), 25th, and 75th percentiles of values, with adjacent values shown with whiskers, and outliers shown as circles. C-GC: intraarticular glucocorticoid cohort; C-IV: intravenous antibiotics cohort; C-PO: oral antibiotics cohort.

In another analysis limited to the first 6 months of treatment, C-IV was associated with a faster rate of resolution than C-PO (HR 1.9, 95\% CI 1.00-3.4; $\mathrm{p}=0.05$ ).

$\mathrm{C}-\mathrm{GC}$ did not differ from $\mathrm{C}-\mathrm{PO}$ in recorded treatment-associated adverse events $[1 / 18(6 \%)$ vs 6/63 (10\%); $\mathrm{p}=0.99]$. In contrast, $\mathrm{C}$-IV experienced a significantly higher prevalence of adverse events [9/31 (29\%); $p=0.03$ vs oral antibiotics], most commonly rash (13\% vs $3 \%$ in C-PO; $6 \%$ in $\mathrm{C}-\mathrm{GC}$ ), headache ( $6 \%$ vs $2 \%$ in C-PO; $0 \%$ in C-GC), fever, and mechanical IV problems (both $6 \%$ vs $0 \%$ in the other groups).

\section{DISCUSSION}

In an observational study of 3 pediatric rheumatology referral centers in a Lyme-endemic region in the United States, we found that second-line IAGC injection, given with or without a concomitant 4-week course of antibiotics, corresponded to a substantially decreased incidence of ARLA and more rapid resolution of arthritis than second-line oral antibiotics alone. These findings were consistent across multiple sensitivity analyses, although some analyses did not reach traditional levels of significance. Documented adverse effects from IAGC (not including procedural pain or bleeding) were similar in frequency to those from oral antibiotics alone and less frequent than adverse effects from IV antibiotics. These findings suggest that IAGC, perhaps in combination with a second course of oral antibiotics, is a potentially effective strategy for second-line treatment. This novel treatment strategy deserves replication and validation in other settings.

While initial therapy for Lyme arthritis has long been supported by clinical trials ${ }^{7,8,17}$, no trials have compared second-line treatments for Lyme arthritis. Thus, the recommendations for second-line treatment in current US treatment guidelines reflect clinical experience and expert guidance. Not surprisingly, clinical practices for second-line treatment are variable. Our study took advantage of this natural practice variation to identify a clinical approach that might lead to more favorable outcomes. While observational comparative effectiveness studies are often fraught with confounding by indication or disease severity, exceptions exist when treatment selection is made based purely on clinical preferences and independent of disease severity ${ }^{18}$. This latter situation appears to have been true in the centers studied, at least with the comparison of IAGC and oral antibiotics alone: there were no measured clinical differences between cohorts except in the use of concomitant treatment (nonsteroidal antiinflammatory drugs were more common for patients who did not receive IAGC). In contrast, children receiving IV antibiotics often had more severe or refractory Lyme arthritis than the other 2 cohorts, making a direct comparison with second-line oral antibiotics more challenging. Compared to oral antibiotics alone, second-line IV antibiotic treatment corresponded to a similar incidence of ARLA. A subset of patients receiving IV therapy may have responded more

Personal non-commercial use only. The Journal of Rheumatology Copyright (C) 2019. All rights reserved. 
quickly than those taking oral antibiotics despite comprising a population with more severe Lyme arthritis. Analyses suggested that those more likely to respond to IV antibiotics may be older children and adolescents, children with shorter duration of joint symptoms before diagnosis, and those with marked worsening (e.g., new joint recruitment) on initial oral antibiotic therapy - hypotheses that bear replication in other settings.

Prior studies have suggested that IAGC preceding the start of antibiotic therapy for Lyme arthritis may predispose patients to ARLA ${ }^{11,13}$. One explanation for this finding is that GC deposited into an infected joint could compromise the immune response that accompanies antibiotic treatment in eradicating intraarticular spirochetes. However, this association between premature IAGC and ARLA has not been shown to be causal and in fact could represent confounding by indication: cases of Lyme arthritis that are inherently more refractory to treatment could also be more likely to receive aggressive treatment before diagnosis with Lyme borreliosis. ARLA itself is thought to represent a postinfectious autoimmune condition ${ }^{19-24}$, a disorder of immune dysregulation $^{25}$, or an aberrant immune reaction to persistent spirochetal antigens within the joint ${ }^{26,27}$. Consistent with the inflammatory features of these purported mechanisms, IAGC injections have been reported as treatments for adults and children with ARLA ${ }^{11,12}$. Our findings suggest that IAGC, perhaps in conjunction with antibiotics, may also have a therapeutic role earlier in the disease course.

While the use of IAGC as second-line therapy for Lyme arthritis may prevent future treatment and promote more rapid resolution of arthritis, it is not a strategy without risk. The procedural risks of IAGC in children are well known ${ }^{28}$. Some patients with Lyme arthritis may respond to a second course of antibiotics alone, and concomitant IAGC may subject them to an unnecessary procedure. In our study, no patient who received second-line IAGC was tested for intraarticular borrelial DNA by PCR. While synovial fluid PCR may be an unreliable marker of active infection in treated patients ${ }^{29}$, second-line IAGC without accompanying antibiotics could theoretically risk prolonged infection in the presence of live, persisting spirochetes. Lyme arthritis does not usually cause permanent joint damage in children and can self-resolve, even if untreated symptoms can persist for years $^{30,31}$. In practice, patients with ARLA may receive a variety of treatments, including (repeated) IAGC injections, disease-modifying drugs, or synovectomy ${ }^{11}$. If early IAGC injection can make additional therapy unnecessary, this approach could benefit patients and avoid further treatment-related harms.

This study had several strengths. We identified a potentially effective, novel approach to managing Lyme arthritis refractory to initial treatment. The magnitude of effects seen with second-line IAGC injections was robust to a variety of different assumptions and modeling conditions in sensitivity analyses. This study was performed in several pediatric referral centers with extensive experience and variable approaches in treating Lyme arthritis.

Our study also had certain limitations. The number of children treated with second-line IAGC was relatively small; our findings - particularly outcomes after second-line IAGC without concomitant antibiotics - should not be considered definitive without replication. It is also unclear whether second-line IAGC might be effective or safe for adults, who are susceptible to more severe, erosive forms of Lyme arthritis $^{32}$. We found no evidence that IAGC were more or less effective based on the age of the child, though our study was not powered to examine this question. This study's referral setting also raises questions about generalizability. However, one cannot expect most general pediatricians to perform IAGC injections, and our findings might argue for earlier specialty referral for children with Lyme arthritis persisting after one antibiotic course. This was an observational study, and it is possible that unmeasured factors related to treatment selection and outcome could explain our results. Nonetheless, the interventional (IAGC) approach related mainly to center of care and physicians' preferences, not measured clinical characteristics. As an observational study, we lack longterm data on patients discharged from clinical followup with either small or no effusions and cannot rule out recurrences in some individuals. Notably, several individuals in each group lacked information on the date of resolution. Assuming that all patients lost to followup had poor outcomes (i.e., ARLA) did not substantively alter our findings. Such an assumption is also probably too strong: Children with a history of arthritis may be more likely not to return to the clinic if they are in remission ${ }^{33}$, particularly after a potentially curative intervention.

Second-line IAGC injection appears to be an effective and safe second-line strategy for persistent Lyme arthritis in children, associated with rapid resolution of symptoms and reduced need for further treatment. Questions remain regarding the role of concomitant antibiotic therapy and the generalizability of these findings, particularly in adult populations. Further research is needed to confirm the effectiveness and safety of this therapeutic approach.

\section{ACKNOWLEDGMENT}

The authors appreciate Jenna Tress's assistance with regulatory documentation and collecting data. The authors also thank Meredith Buckley, Kelly Collier, Janille Diaz, Elizabeth Kaufman, Valerie Levy, Bernadette Lewcun, and Amanda Schlefman for data collection.

\section{ONLINE SUPPLEMENT}

Supplementary material accompanies the online version of this article.

\section{REFERENCES}

1. Kugeler KJ, Farley GM, Forrester JD, Mead PS. Geographic distribution and expansion of human Lyme disease, United States. Emerg Infect Dis 2015;21:1455-7. 
2. Rosenberg R, Lindsey NP, Fischer M, Gregory CJ, Hinckley AF, Mead PS, et al. Vital signs: trends in reported vectorborne disease cases - United States and territories, 2004-2016. MMWR Morb Mortal Wkly Rep 2018;67:496-501.

3. Hubalek Z. Epidemiology of Lyme borreliosis. Curr Probl Dermatol 2009;37:31-50.

4. Steere AC, Schoen RT, Taylor E. The clinical evolution of Lyme arthritis. Ann Intern Med 1987;107:725-31.

5. Gerber MA, Zemel LS, Shapiro ED. Lyme arthritis in children: clinical epidemiology and long-term outcomes. Pediatrics 1998; 102:905-8.

6. Steere AC, Glickstein L. Elucidation of Lyme arthritis. Nat Rev Immunol 2004;4:143-52.

7. Steere AC, Levin RE, Molloy PJ, Kalish RA, Abraham JH, Liu NY, et al. Treatment of Lyme arthritis. Arthritis Rheum 1994;37:878-88.

8. Dattwyler RJ, Wormser GP, Rush TJ, Finkel MF, Schoen RT, Grunwaldt E, et al. A comparison of two treatment regimens of ceftriaxone in late Lyme disease. Wien Klin Wochenschr 2005; 117:393-7.

9. Wormser GP, Dattwyler RJ, Shapiro ED, Halperin JJ, Steere AC, Klempner MS, et al. The clinical assessment, treatment, and prevention of Lyme disease, human granulocytic anaplasmosis, and babesiosis: clinical practice guidelines by the Infectious Diseases Society of America. Clin Infect Dis 2006;43:1089-134.

10. Lyme disease. In: Kimberlin DW, Brady MT, Jackson MA, Long SS, editors. Red book: 2015 report of the Committee on Infectious Diseases. Itasca, IL: American Academy of Pediatrics; 2015:516-25.

11. Steere AC, Angelis SM. Therapy for Lyme arthritis: strategies for the treatment of antibiotic-refractory arthritis. Arthritis Rheum 2006;54:3079-86.

12. Nimmrich S, Becker I, Horneff G. Intraarticular corticosteroids in refractory childhood Lyme arthritis. Rheumatol Int 2014;34:987-94.

13. Huppertz HI, Karch H, Suschke HJ, Döring E, Ganser G, Thon A, et al. Lyme arthritis in European children and adolescents. The Pediatric Rheumatology Collaborative Group. Arthritis Rheum 1995;38:361-8.

14. Horton DB, Taxter AJ, Davidow AL, Groh B, Sherry DD, Rosé CD. Pediatric antibiotic-refractory Lyme arthritis: a multicenter case-control study. J Rheumatol 2019 Mar 1 (E-pub ahead of print).

15. Harris PA, Taylor R, Thielke R, Payne J, Gonzalez N, Conde JG. Research electronic data capture (REDCap) - a metadata-driven methodology and workflow process for providing translational research informatics support. J Biomed Inform 2009;42:377-81.

16. Sterne JA, White IR, Carlin JB, Spratt M, Royston P, Kenward MG, et al. Multiple imputation for missing data in epidemiological and clinical research: potential and pitfalls. BMJ 2009;338:b2393.

17. Dattwyler RJ, Halperin JJ, Volkman DJ, Luft BJ. Treatment of late Lyme borreliosis - randomised comparison of ceftriaxone and penicillin. Lancet 1988;1:1191-4.

18. Strom BL, Melmon KL. The use of pharmacoepidemiology to study beneficial drug effects. In: Strom BL, Kimmel SE, Hennessy S, editors. Pharmacoepidemiology, fifth ed. West Sussex: Wiley; 2011:655-77.
19. Steere AC, Dwyer E, Winchester R. Association of chronic Lyme arthritis with HLA-DR4 and HLA-DR2 alleles. N Engl J Med 1990;323:219-23.

20. Kalish RA, Leong JM, Steere AC. Association of treatment-resistant chronic Lyme arthritis with HLA-DR4 and antibody reactivity to OspA and OspB of Borrelia burgdorferi. Infect Immun 1993;61:2774-9.

21. Londoño D, Cadavid D, Drouin EE, Strle K, McHugh G, Aversa $\mathrm{JM}$, et al. Antibodies to endothelial cell growth factor and obliterative microvascular lesions in the synovium of patients with antibiotic-refractory Lyme arthritis. Arthritis Rheumatol 2014;66:2124-33

22. Shen S, Shin JJ, Strle K, McHugh G, Li X, Glickstein LJ, et al. Treg cell numbers and function in patients with antibiotic-refractory or antibiotic-responsive Lyme arthritis. Arthritis Rheum 2010; 62:2127-37.

23. Vudattu NK, Strle K, Steere AC, Drouin EE. Dysregulation of $\mathrm{CD} 4+\mathrm{CD} 25$ (high) $\mathrm{T}$ cells in the synovial fluid of patients with antibiotic-refractory Lyme arthritis. Arthritis Rheum 2013; 65:1643-53.

24. Strle K, Sulka KB, Pianta A, Crowley JT, Arvikar SL, Anselmo A, et al. T-helper 17 cell cytokine responses in Lyme disease correlate with Borrelia burgdorferi antibodies during early infection and with autoantibodies late in the illness in patients with antibiotic-refractory Lyme arthritis. Clin Infect Dis 2017;64:930-8.

25. Lochhead RB, Strle K, Kim ND, Kohler MJ, Arvikar SL, Aversa $\mathrm{JM}$, et al. MicroRNA expression shows inflammatory dysregulation and tumor-like proliferative responses in joints of patients with postinfectious Lyme arthritis. Arthritis Rheumatol 2017;69:1100-10.

26. Bockenstedt LK, Gonzalez DG, Haberman AM, Belperron AA. Spirochete antigens persist near cartilage after murine Lyme borreliosis therapy. J Clin Invest 2012;122:2652-60.

27. Wormser GP, Nadelman RB, Schwartz I. The amber theory of Lyme arthritis: initial description and clinical implications. Clin Rheumatol 2012;31:989-94.

28. Ravelli A, Lattanzi B, Consolaro A, Martini A. Glucocorticoids in paediatric rheumatology. Clinical Exp Rheumatol 2011;5 Suppl 68:S148-52.

29. Li X, McHugh GA, Damle N, Sikand VK, Glickstein L, Steere AC. Burden and viability of Borrelia burgdorferi in skin and joints of patients with erythema migrans or Lyme arthritis. Arthritis Rheum 2011;63:2238-47.

30. Steere AC, Schoen RT, Taylor E. The clinical evolution of Lyme arthritis. Ann Intern Med 1987:107:725-31.

31. Szer IS, Taylor E, Steere AC. The long-term course of Lyme arthritis in children. N Engl J Med 1991;325:159-63.

32. Lawson JP, Steere AC. Lyme arthritis: radiologic findings. Radiology 1985;154:37-43.

33. Fantini F, Gerloni V, Gattinara M, Cimaz R, Arnoldi C, Lupi E. Remission in juvenile chronic arthritis: a cohort study of 683 consecutive cases with a mean 10 year followup. J Rheumatol 2003;30:579-84. 